\title{
Piperine Exhibits Promising Antibiofilm Activity Against Staphylococcus Aureus by Accumulating Reactive Oxygen Species (ROS)
}

\section{Sharmistha Das}

The Neotia University

Payel Paul

The Neotia University

\section{Sudipta Chatterjee}

The Neotia University

\section{Poulomi Chakraborty}

The Neotia University

\section{Ranojit K. Sarker}

The Neotia University

\section{Amlan Das}

The Neotia University

\section{Debasish Maiti}

\section{Tripura University}

Prosun Tribedi ( $\nabla$ tribedi.prosun@gmail.com )

The Neotia University https://orcid.org/0000-0002-6567-006X

\section{Research Article}

Keywords: Biofilm, Staphylococcus aureus, Piperine, Antibiofilm, Reactive oxygen species

Posted Date: May 13th, 2021

DOI: https://doi.org/10.21203/rs.3.rs-514513/v1

License: (c) (1) This work is licensed under a Creative Commons Attribution 4.0 International License. Read Full License

Version of Record: A version of this preprint was published at Archives of Microbiology on December 23rd, 2021. See the published version at https://doi.org/10.1007/s00203-021-02642-7. 


\section{Abstract}

Biofilm, an aggregated form of microbial existence has been a major area of concern in the healthcare units. These sessile microbes not only protect themselves from the host immune system but also exhibit high resistance against several antimicrobials. One such widely reported Gram-positive pathogen is Staphylococcus aureus. This human commensal is known to cause severe harmful diseases like bacteremia, sepsis, pneumonia, etc. Thus, strategies need to be undertaken to deal with such biofilm challenges. In this respect, we aimed to inhibit microbial biofilm formation of Staphylococcus aureus under the influence of a natural compound, piperine. Our study revealed that the higher concentrations of piperine exhibited considerable antimicrobial activity against Staphylococcus aureus. Hence, lower concentrations of piperine were tested to examine its antibiofilm activity. Several experiments like crystal violet (CV) assay, total biofilm protein assay, and fluorescence microscopy observation established that lower concentrations ( $8 \mu \mathrm{g} / \mathrm{mL}$ and $16 \mu \mathrm{g} / \mathrm{mL}$ ) of piperine showed efficient antibiofilm activity against Staphylococcus aureus. It was also noticed that the lower concentrations of piperine did not compromise the microbial growth of Staphylococcus aureus while exhibiting antibiofilm activity. In this connection, we also noticed that the lower concentrations of piperine showed a considerable reduction in microbial metabolic activity. Furthermore, we observed that the compound was found to accumulate reactive oxygen species in the bacterial cells that could play an important role in the inhibition of biofilm formation. Thus, piperine could be considered as a potential antibiofilm agent against the biofilm formation caused by Staphylococcus aureus.

\section{Introduction}

Microorganisms are seen to exist in nature in two forms, one is known as the free-living planktonic cells, other is termed as the biofilm (Gupta et al. 2016; Chakraborty et al. 2018a). Bacterial biofilm is the aggregation of either homogeneous or heterogeneous conformations of bacteria on a biotic or abiotic surface (Hurlow et al. 2015; Gupta et al. 2016). In this manner, the bacterial aggregates form a matured cluster by producing a self-secreted extracellular polymeric substance. This matrix was found to obstruct the movement of antibiotics thereby limiting the action of such medicines (Cortes et al. 2011). Biofilms have been reported to be 1000 times resistant to antibiotics in contrast to their planktonic form (Gupta et al. 2016; Paul et al. 2020). As per the literature survey, nearly $80 \%$ of bacterial infections are linked to biofilm (Davies 2003). Staphylococcus aureus is one such well-known biofilm-forming Gram-positive bacteria. This bacterium is a human commensal which under certain circumstances can also act as an opportunistic pathogen consequently leading to the spread of pathogenesis (Das et al. 2016). Staphylococcus aureus is linked to several chronic infections like wound infections, boils, cellulitis, folliculitis, pneumonia, osteomyelitis, meningitis, and so on (Moreira et al. 2017). A major concern of the present times is to deal with these biofilm challenges and come up with effective strategies. In this regard, several natural and synthetic molecules have been examined to understand their antibiofilm activity against various biofilm-forming organisms (Das et al. 2016; Chakraborty et al. 2018b; Chakraborty et al. 2020). Since natural molecules are cost-effective and more beneficial than synthetic 
ones, they have been paid more attention to biofilm inhibition studies. Moreover, among the naturally occurring alkaloids, the compound piperine (a bio-active component of pepper) has gained much interest in recent reports for its diverse biological applications (antimicrobial, antiparasitic, anticancer effect, and other pharmacological effects) (Quijia and Chorilli 2020). However, its antibiofilm activity is still under investigation. Thus, for our present study, we have considered studying the antibiofilm effect of piperine against the biofilm-forming organism, Staphylococcus aureus. Our experimental analysis revealed that piperine could be considered for the effective management of biofilm threats.

\section{Materials And Methods}

\section{Microbial strain, growth media, and culture conditions}

The test organism considered for the present study was Staphylococcus aureus (MTCC 96). This Grampositive bacterium was offered to us as a kind gift from Dr. Surajit Bhattacharjee (Assistant Professor, Department of Molecular Biology and Bioinformatics, Tripura University, India). Luria-Bertani (LB) media purchased from Himedia, India, was used for securing the optimum growth of the test organism. Staphylococcus aureus was grown at $37^{\circ} \mathrm{C}$ as per the requirement of the experiments. The test compound, piperine used for the present study was given to us by Dr. Amlan Das (Department of Chemistry, NIT Sikkim, India). Piperine was dissolved into an organic solvent, dimethyl sulfoxide (DMSO) to prepare its stock solution. Thereafter, the piperine solution was further transferred to sterile LB broth as per the requirement of the study.

\section{Assessment of microbial viability}

The antimicrobial effect of piperine against Staphylococcus aureus was estimated by colony forming units (CFU) method. To test the same, an equal number of Staphylococcus aureus $\left(1 \times 10^{5} \mathrm{CFU} / \mathrm{mL}\right.$ ) was inoculated in freshly autoclaved LB media. To it, varying concentrations $(250,500,750$, and $1000 \mu \mathrm{g} / \mathrm{mL})$ of piperine were aseptically incorporated in the respective tubes. Additionally, a control set was also maintained in which only the test organism was added without supplementing it with piperine. All the experimental sets were further incubated at $37^{\circ} \mathrm{C}$ for $24 \mathrm{~h}$. Post incubation, an equal volume of microbial cultures was collected from each growth media and serially diluted with $0.85 \% \mathrm{NaCl}$ solution. After that, the microbial viability in each experimental set was determined by measuring the CFU count assay as described by Chakraborty et al. (2018a).

\section{Assessment of microbial biofilm formation}

Previous literature revealed that the Crystal violet (CV) assay could be used to determine the extent of microbial biofilm formation under different conditions (Mukherjee et al. 2013). To measure the biofilm formation of Staphylococcus aureus under the influence of piperine, an equal number of organisms $\left(1 \times 10^{5} \mathrm{CFU} / \mathrm{mL}\right)$ were separately inoculated in several test tubes containing $5 \mathrm{~mL}$ of sterile LB media. To it, different concentrations ( 8 and $16 \mu \mathrm{g} / \mathrm{mL}$ ) of piperine were separately added. However, in the control set, a similar number of organisms were grown without exposing it to piperine. All the growth media 
including the control were then incubated at $37^{\circ} \mathrm{Cfor} 24 \mathrm{~h}$. After the incubation, the planktonic cells were removed from each set followed by washing the tubes with sterile double distilled water. The tubes were further air-dried and exposed to $5 \mathrm{~mL}$ of $(0.4 \%) \mathrm{CV}$ solution for $30 \mathrm{~min}$. Then, the CV solution was discarded from all the tubes and washed with sterile double distilled water. To estimate the degree of microbial biofilm formation, the CV-stained biofilm cells were dissolved with $33 \%$ glacial acetic acid and its absorbance was estimated at $630 \mathrm{~nm}$.

\section{Assessment of biofilm protein}

The determination of total biofilm protein could be considered as an indirect estimation of the degree of microbial colonization (Tribedi et al. 2015). The previous literature recommended that there has been a directly proportional relationship between the extent of protein recovery and the degree of microbial colonization (Tribedi et al. 2015; Paul et al. 2020). A greater protein recovery could be expected from a dense microbial association and vice versa. Thus, to measure the total biofilm protein count both in the

presence and absence of the test compound, a similar number of cells $\left(1 \times 10^{5} \mathrm{CFU} / \mathrm{mL}\right)$ were added into several test tubes containing sterile LB media. A control set was also taken into consideration in which the cells were grown without any exposure to piperine. All the test tubes were then incubated at $37^{\circ} \mathrm{C}$ for $24 \mathrm{~h}$. After the incubation, the planktonic cells were discarded from all the test tubes. Next, the tubes were washed, adequately dried and $5 \mathrm{~mL}$ of $0.3(\mathrm{M}) \mathrm{NaOH}$ was added to it. After boiling the tubes at $100^{\circ} \mathrm{C}$ for $30 \mathrm{~min}$, the respective suspensions in each tube were subjected to centrifugation for $10 \mathrm{~min}$ at $8000 \mathrm{rpm}$. Afterward, the supernatant was recovered from each tube and the protein content of the supernatant was measured by following the protocol of Lowry et al. (1951).

\section{Analysis of microbial colonization}

The degree of microbial colonization under different conditions could be observed under a fluorescence microscope as mentioned by Paul et al. (2020). Thus, to test the effect of piperine on microbial colonization, an equal number of cells $\left(1 \times 10^{5} \mathrm{CFU} / \mathrm{mL}\right)$ were inoculated in $5 \mathrm{~mL}$ of sterile LB media challenged with different concentrations ( 8 and $16 \mu \mathrm{g} / \mathrm{mL}$ ) of piperine. Alongside, a control set was also kept in which a similar number of the organism was grown in the absence of piperine. To all the experimental sets, sterile cover-slips were incorporated aseptically. After that, all the growth media including the control was incubated at $37^{\circ} \mathrm{C}$ for $24 \mathrm{~h}$. Post incubation, the cover-slips were recovered carefully and stained with acridine orange $(4 \mu \mathrm{g} / \mathrm{mL})$. After that, the stained coverslips were observed under a fluorescence microscope to observe the degree of microbial colonization under the given conditions.

\section{Assessment of antimicrobial activity}

To examine the antimicrobial effect of piperine against Staphylococcus aureus, a series of experiments were performed. At first, the pattern of microbial growth curve was compared between the piperine treated and untreated conditions. To do the test, an equal number of the cells $\left(1 \times 10^{5} \mathrm{CFU} / \mathrm{mL}\right)$ were inoculated 
into different conical flasks carrying $100 \mathrm{~mL}$ of autoclaved LB media. To it, varying concentrations (8 and $16 \mu \mathrm{g} / \mathrm{mL}$ ) of piperine were separately added. However, in the control set, a similar number of cells were grown in the sterile LB without exposing it to piperine. All the growth media were then incubated at $37^{\circ} \mathrm{C}$ for $24 \mathrm{~h}$. At different time intervals, microbial cultures were recovered from each growth media and the optical density of the same was recorded through a spectrophotometer at $600 \mathrm{~nm}$. However, in the second experiment, the microbial cultures $(1 \mathrm{~mL})$ were separately collected from both piperine treated and untreated growth media after $24 \mathrm{~h}$ of incubation at $37^{\circ} \mathrm{C}$. The collected cultures were serially diluted with $9 \mathrm{~mL}$ of autoclaved $0.85 \% \mathrm{NaCl}$ solution. Post dilution, the aliquots $(100 \mu \mathrm{L})$ from different dilutions of both piperine treated and untreated growth media were separately spread on sterile LB agar plates and incubated at $37^{\circ} \mathrm{C}$ for another $24 \mathrm{~h}$. After the incubation, the colony-forming unit (CFU) of each sample was calculated by following the protocol mentioned by Paul et al. (2020). Besides, a spot assay was also performed in which the dilutions $\left(10^{-1}\right.$ to $\left.10^{-5}\right)$ prepared from both piperine treated and untreated growth media were separately spotted on the sterile LB agar plate. Again, the plate was incubated at $37^{\circ} \mathrm{C}$ for 24 h. Post incubation, the developed microbial spots corresponding to each dilution were analyzed. To gain further confidence, the clear zone assay was performed to test the antimicrobial effect of piperine against Staphylococcus aureus. To do the test, firstly, $100 \mu \mathrm{L}$ culture of Staphylococcus aureus was spread over the LB agar plate to prepare the microbial lawn over the plate. Thereafter, three discrete wells were punctured on the LB agar plates and each well was loaded with the tested concentrations (8 and 16 $\mu \mathrm{g} / \mathrm{mL}$ ) of piperine. One of the wells was considered as the control in which instead of piperine DMSO was applied. Plates were then incubated at $37^{\circ} \mathrm{C}$ for $24 \mathrm{~h}$ to observe the clear zone (if any) around the punctured wells.

\section{Assessment of metabolic activity}

The metabolic activity of the test organism was estimated by adhering to the protocol of Fluorescein diacetate (FDA) hydrolysis assay as stated by Chakraborty and Tribedi (2019). To examine the metabolic activity of Staphylococcus aureus under the presence and absence of piperine, a similar number of the organism $\left(1 \times 10^{5} \mathrm{CFU} / \mathrm{mL}\right)$ were inoculated into various test tubes containing sterile LB media. After that, the cells in the tubes were either challenged to piperine or left unexposed. All the tubes were incubated for $24 \mathrm{~h}$ at $37^{\circ} \mathrm{C}$. After the incubation, planktonic cells were discarded from all the tubes. Thereafter, the tubes were washed with sterile Milli-Q water and air-dried adequately. Then, $5 \mathrm{~mL}(60 \mathrm{mM})$ phosphate buffer ( $\mathrm{pH}$ of 7.6) was added to each tube and gently vortexed. Afterward, FDA (10 mg/mL) dissolved in acetone was added to each tube and further incubated for $1 \mathrm{~h}$ at $37^{\circ} \mathrm{C}$. Post incubation, the tubes were centrifuged at $10000 \mathrm{rpm}$ for $10 \mathrm{~min}$. After that, the supernatant was collected from each tube and the absorbance of the same was recorded at $490 \mathrm{~nm}$.

\section{Measurement of cellular accumulation of reactive oxygen species (ROS)}

To determine the cellular accumulation of ROS under different conditions, a DCFDA (2',7'-

dichlorofluorescein diacetate) based ROS detection assay kit (ab113851) was practiced in the present study. Fluorescent DCFDA upon entering into the microorganisms gets deacetylated by the microbial 
esterase to a non-fluorescent molecule $\left\{2^{\prime}, 7^{\prime}\right.$-dichlorofluorescein (DCF) $\}$. The cellular accumulation of ROS can further oxidize this DCF to a fluorogenic DCF which can be estimated with the help of a fluorescence spectrophotometer at an excitation and emission wavelength of $488 \mathrm{~nm}$ and $535 \mathrm{~nm}$, respectively. To determine the effect of piperine on the cellular accumulation of ROS, DCFDA was added to both piperine treated and untreated cells $\left(1 \times 10^{8} \mathrm{CFU} / \mathrm{mL}\right)$ followed by the incubation at $37^{\circ} \mathrm{C}$ for $30 \mathrm{~min}$. After the incubation, the DCFDA exposed cells were centrifuged at $8000 \mathrm{rpm}$ for $10 \mathrm{~min}$. Thereafter, the pellet was collected, washed with sterile LB, and subsequently exposed to different concentrations (8 and 16 $\mu \mathrm{g} / \mathrm{mL}$ ) of piperine. To validate our results, an antioxidant (ascorbic acid) was also incorporated in the cells which were previously exposed to the varying concentrations of piperine. A control set was also kept which contained only the microbial cells. Neither piperine nor ascorbic acid was applied to the cells in the control set. Finally, the extent of the production of fluorogenic DCF was estimated by a fluorescence spectrophotometer (Dwivedi et al. 2014).

\section{Assessment of the microbial motility}

Literature reports stated that the motility of Staphylococcus aureus could be analyzed over soft agar media (Kaito and Sekimizu 2007; Tsompanidou et al. 2011; Paul et al. 2020). To test the same, a semisolid agar media was prepared ( $30 \mathrm{~g} / \mathrm{L}$ tryptone soya broth along with $5 \mathrm{~g} / \mathrm{L}$ of glucose and $8 \mathrm{~g} / \mathrm{L}$ of agar), autoclaved, and poured in sterile Petri plates. After that, an equal number of cells was separately recovered from both piperine treated and untreated growth media and spotted at the center of each Petri plate. Following this, the plates were kept aside under aseptic conditions for $30 \mathrm{~min}$ to allow the microbial spots to dry. Afterward, the plates were incubated at $37^{\circ} \mathrm{C}$ for $24 \mathrm{~h}$. Also, the motility on each plate was estimated by measuring the diameter (in $\mathrm{mm}$ ) from the point of spot inoculation (Kumar et al. 2013).

\section{Statistical Analysis}

One-way analysis of variance (ANOVA) was used to analyze all the statistical tests of our current study. The mean value was presented after repeating each experiment three times. Error bars indicated standard deviations. The $\mathrm{P}$ values incorporated specify the measure of significance in contrast to the control. $\mathrm{P}$ values $<0.05$ marked with $(*),<0.01$ marked with $(* *)$, and $<0.001$ marked with $(* \star *)$ in comparison to the control. P values that were above 0.05 were marked as N.S. (no statistical difference).

\section{Results And Discussion}

Higher concentrations of piperine exhibited efficient antimicrobial activity against Staphylococcus aureus

Since alkaloids were reported to show different biological activities including antimicrobial properties (Cowan 1999), in the present study, antimicrobial activity of piperine was estimated against Staphylococcus aureus by determining the colony-forming units (CFU). To determine the viable microbial counts of Staphylococcus aureus under the influence of piperine, an equal number of the organism was inoculated into sterile LB media in which varying concentrations of piperine were separately added. A control set was also made wherein the organism was grown in the absence of piperine. All the treated 
and control growth media were incubated for $24 \mathrm{~h}$ at $37^{\circ} \mathrm{C}$. Post incubation, it was observed that the control set showed the highest number of CFU count. The result also revealed a notable decrease in the CFU count in the presence of the test compound (Figure 1). The present report suggested that at the highest concentration $(1000 \mu \mathrm{g} / \mathrm{mL})$ of piperine, the lowest number of viable colonies were estimated thereby indicating an inversely proportional relationship between the concentrations of the compound and microbial viability (Figure 1). Thus, the result revealed the notable antimicrobial activity of piperine against Staphylococcus aureus at its high concentrations.

\section{Piperine exhibited efficient antibiofilm activity against Staphylococcus aureus}

Literature survey highlights the major challenges of healthcare units linked to biofilm infections (Dewasthale et al. 2018). It has also been noted that bacterial infections linked to biofilm have been found to promote pathogenicity considerably (Cortes et al. 2011). Moreover, the drug resistance property of such biofilm aggregates is a major area of concern and thus demands the need for urgent and effective strategies to inhibit them (Crossley et al. 2009). In this direction, to study the effect of the test compound, piperine, on the biofilm profile of Staphylococcus aureus, crystal violet (CV) assay was carried out by following the protocol of Mukherjee et al. (2013). The result revealed that in the control set where the test compound was not added, maximum biofilm formation was seen to take place (Figure 2A). On the other hand, a gradual increase in the concentrations of piperine revealed a significant decrease in the biofilm formation(Figure 2A). Further, the result indicated that piperine $(16 \mu \mathrm{g} / \mathrm{mL})$ exhibited the highest inhibition in biofilm formation of Staphylococcus aureus (Figure 2A). To reassure the CV assay results, the total protein count of the adhered microbial cells was also estimated as the proteins originate from those microorganisms thereby establishing a directly proportional relationship between the degree of microbial associations and the extent of protein recovery. Thus, to compare the microbial associations between the piperine treated and untreated growth media, an equal number of microbial cells of Staphylococcus aureus was inoculated both in the presence and absence of piperine. Then, all the sets were incubated at $37^{\circ} \mathrm{C}$ for $24 \mathrm{~h}$. Post incubation, the highest protein recovery was found to take place in the control set, which was not exposed to piperine (Figure 2B). However, the protein count decreased significantly with the gradual increase in the concentration of the test compound (Figure 2B). The protein recovery was found to be the lowest when the cells were treated with $16 \mu \mathrm{g} / \mathrm{mL}$ of piperine (Figure 2B). Thus, from CV assay and protein recovery data, it could be stated that the tested concentrations of piperine significantly inhibited the biofilm formation of Staphylococcus aureus. Besides, a microscopic observation was also undertaken to further confirm the antibiofilm activity of piperine against Staphylococcus aureus. To do so, a nearly equal number of cells were grown in sterile LB under the presence and absence of piperine. Sterile coverslips were separately added to each growth media so that the organism could form biofilm over the coverslip surface. After an incubation of $24 \mathrm{~h}$ at $37^{\circ} \mathrm{C}$, coverslips were taken out from each growth media, stained with acridine orange, and observed under a fluorescence microscope. A significant variation in microbial biofilm population was observed among the coverslips taken from each growth media. We observed the maximum biofilm aggregates on the coverslips which were not exposed to piperine (Figure $2 \mathrm{C}$ ). We also noticed that the biofilm clusters got reduced 
considerably when the cells were exposed to piperine (Figure 2C). Taken together, all the results demonstrated that the tested concentrations of piperine exhibited efficient antibiofilm activity against Staphylococcus aureus.

\section{The tested concentrations of piperine did not show any considerable antimicrobial activity against Staphylococcus aureus}

The current study aims to come up with an effective antibiofilm agent which does not show any antimicrobial property. Though we have observed that the lower concentrations $(8 \mu \mathrm{g} / \mathrm{mL}$ and $16 \mu \mathrm{g} / \mathrm{mL}$ ) of piperine exhibited efficient antibiofilm activities, however, the antimicrobial effect of the same is yet to be examined against Staphylococcus aureus. Thus, the microbial growth profile of Staphylococcus aureus was examined under the presence and absence of piperine. To do the same, an equal number of the organism was separately inoculated in $100 \mathrm{~mL}$ of sterile LB media. Similarly, a control set was also prepared where the cells remained unexposed to piperine. All the growth media were further incubated at $37^{\circ} \mathrm{C}$ for a period of $24 \mathrm{~h}$. At different time intervals, an equal volume of microbial culture was individually recovered from each set (control and treated) and its absorbance was recorded at $600 \mathrm{~nm}$. The observations exhibited no substantial variation in the microbial growth pattern in either of the experimental sets (Figure 3A). Thus, the result of the same indicated that the growth profile of Staphylococcus aureus remained unaffected due to piperine exposure. To validate our results further, viable microbial counts were also determined in both piperine treated and untreated growth media by following the CFU method (Chakraborty et al. 2018a). The result showed that the number of microbial colonies estimated was almost the same for all the experimental sets (Figure 3B). The result further indicated that the tested concentrations $(8 \mu \mathrm{g} / \mathrm{mL}$ and $16 \mu \mathrm{g} / \mathrm{mL})$ of piperine did not show any antimicrobial action against Staphylococcus aureus. In support of our observations, spot assay and clear zone assays were also performed to reassure the antimicrobial effect of piperine (if any) against Staphylococcus aureus. In the case of spot assay, the diluted samples collected from both piperine treated and untreated cultures were spotted on sterile LB agar plates and incubated at $37^{\circ} \mathrm{C}$ for $24 \mathrm{~h}$. After the incubation, the pattern of the developed microbial spots was analyzed. The result showed that no considerable variation in microbial growth was spotted between piperine-treated and untreated cultures (Figure 3C). Thus, the result again indicated that the used concentrations $(8 \mu \mathrm{g} / \mathrm{mL}$ and $16 \mu \mathrm{g} / \mathrm{mL})$ of piperine did not exhibit any antimicrobial activity against Staphylococcus aureus. Moreover, for the clear zone assay, the tested concentrations of piperine $(0 \mu \mathrm{g} / \mathrm{mL}, 8 \mu \mathrm{g} / \mathrm{mL}$, and $16 \mu \mathrm{g} / \mathrm{mL})$ were placed in the wells of the microbial lawn prepared on LB agar plate. After an incubation of $24 \mathrm{~h}$ at $37^{\circ} \mathrm{C}$, the clear zone around the well (if any) was examined. Post incubation, no zone of inhibition was observed around any of the punctured wells on the LB agar plates (Figure 3D). Thus, the results demonstrated that the used concentrations of piperine for the biofilm inhibition studies did not exhibit any antimicrobial activities against Staphylococcus aureus.

\section{The tested concentrations of piperine exhibited a considerable reduction in the microbial metabolic activity}


Microorganisms in biofilm secrete protease enzyme during the evasion to the host tissue (Chakraborty and Tribedi 2019). Thus, in the current study, efforts have been made to analyze whether the test compound could reduce the microbial metabolic activity of Staphylococcus aureus. The metabolic activity of the organisms under the presence and absence of piperine was determined by FDA hydrolysis assay under similar conditions. To do the same, an equal number of Staphylococcus aureus cells were either exposed to piperine or left unexposed to piperine. Later, all the experimental sets were incubated at $37^{\circ} \mathrm{C}$ for $24 \mathrm{~h}$. After the completion of the incubation, the planktonic cells were discarded and the metabolic activity of the adhered biofilm cells was measured by following the FDA assay. The result of the FDA hydrolysis assay showed that the metabolic activity of piperine exposed cells was significantly less in contrast to the unexposed control set (Figure 4). The data also revealed that the maximum metabolic activity was shown by the cells which were not exposed to piperine (Figure 4). Thus, the result indicated that the selected concentrations of the compound $(8 \mu \mathrm{g} / \mathrm{mL}$ and $16 \mu \mathrm{g} / \mathrm{mL})$ were found to reduce the metabolic activity of the organism efficiently. The results so far also suggested that the compound exhibited biofilm inhibition as well as a reduction in metabolic activity of Staphylococcus aureus efficiently.

\section{The tested concentrations of piperine showed considerable accumulation of cellular ROS in Staphylococcus aureus}

Reactive oxygen species include highly reactive molecules formed by the inappropriate transferring of electrons to $\mathrm{O}_{2}$. Peroxides, hydroxyl radical, singlet oxygen, superoxide, etc. belong to the components of ROS(Bergamini et al. 2004). Previous literature reported that the generation of ROS could be considered as one of the potential causes of biofilm inhibition (Dwivedi et al. 2014; Paul et al. 2020). Thus, in the present study, the cellular accumulation of ROS under the presence and absence of the compound (piperine) was examined by following the DCFDA assay. The result revealed a significant difference in the ROS profile between the piperine treated and untreated cells (Figure $5 \mathrm{~A}$ ). It is noticeable from the results that the control set, which was not exposed to any concentration of piperine showed the lowest accumulation of ROS, whereas the same gradually increased with the increase in the piperine concentration (Figure 5A). The results further showed that the maximum ROS accumulation was seen to take place at a piperine concentration of $16 \mu \mathrm{g} / \mathrm{mL}$ (Figure 5A). To further validate our results, ascorbic acid was taken into consideration as it has been reported as a potential antioxidant molecule (Fukumura et al. 2012). To test the role of ascorbic acid on the cellular accumulation of ROS, a varying concentration of ascorbic acid was added to the cells which were previously exposed to piperine. The result showed that the ROS which was accumulated due to piperine treatment got reduced considerably under the exposure of ascorbic acid (Figure 5A). Thus, the result indicated that the inhibition in biofilm formation of Staphylococcus aureus could be attributed to the cellular accumulation of ROS. To understand the effect of ROS accumulation on biofilm inhibition, several experiments were carried out wherein in the first set, cells were neither exposed to piperine nor ascorbic acid. In the second set, a similar number of cells were exposed only to piperine. However, in the third set, a similar number of cells were exposed to both piperine and ascorbic acid. All the sets were incubated at $37^{\circ} \mathrm{C}$ for $24 \mathrm{~h}$. After the incubation, the extent of 
microbial biofilm formation was examined in each experimental set. The result showed that the control set which was neither exposed to piperine nor ascorbic acid exhibited the maximum biofilm formation (Figure 5B). However, the extent of biofilm formation got considerably inhibited when the cells were exposed to piperine (Figure 5B). The result also revealed that ascorbic acid treatment was found to restore the biofilm formation ability of Staphylococcus aureus efficiently (Figure 5B). Thus, the results suggested that piperine-treated cells accelerated the generation of ROS in Staphylococcus aureus which in turn led to a significant inhibition in biofilm formation of the bacteria.

\section{The tested concentrations of piperine exhibited considerable inhibition in microbial motility}

Microbial motility happens to be a key factor for the promotion of biofilm formation as it considerably enhances microbial colonization to various surfaces (Merritt et al. 2007). The existing literature reported that microbial motility is regulated by quorum sensing (Daniels et al. 2004). Quorum sensing is a densitydependent phenomenon in which the microorganism exhibits various functions including microbial motility after reaching the threshold density (Chakraborty et al. 2020). Thus, in the present study, the effects of piperine on the quorum sensing property of Staphylococcus aureus were tested by targeting microbial motility. Hence, to understand the influence of piperine on the microbial motility of Staphylococcus aureus, an equal number of cells were independently collected from both piperine treated and untreated growth media and spotted accordingly at the center of the plate. Post incubation, the result revealed that the culture media that was not treated with piperine showed the highest motility (Figure 6A). On contrary, the cells which were exposed to piperine showed reduced motility in comparison to the control (Figure 6A). Thus, the result indicated that the increase in the concentrations of piperine could decrease microbial motility significantly. To support this observation further, the zone of diameter (in $\mathrm{mm}$ ) of microbial motility was also measured from the center of the culture plates (Figure 6B). The result showed that the highest diameter of microbial motility was found for the experimental set which was not exposed to piperine (Figure 6B). However, the diameter of microbial motility decreased under the exposure of the tested concentrations of the compound (Figure 6B). Thus, the results revealed that the tested concentrations of piperine interfered with the quorum sensing property of the organism by inhibiting its motility property.

\section{Conclusion}

The alarming rise in biofilm-linked infections is a major area of concern in recent times in the field of public healthcare. The high resistance of biofilm aggregates against several antimicrobial agents calls for urgent attention in the scientific world. Thus, effective strategies need to be adopted in this direction. From this study, it appears that a natural compound, piperine, could act as a potential antibiofilm agent against the biofilm formation of Staphylococcus aureus by accumulating ROS. Thus, this natural compound could be used alone or in combination with other potential antibiofilm agents to curb the biofilm challenges.

\section{Declarations}




\section{Funding}

Not applicable

\section{Conflicts of interest}

The authors have no conflicts of interest to declare that are relevant to the content of this article.

\section{Availability of data and material}

The datasets generated during and/or analyzed during the current study are available from the corresponding author on reasonable request.

\section{Code availability}

Two software namely Minitab 19 and NCSS were used for the current study. Both the software happened to be the trail version. And we were allowed to access the same for a period of 30 days.

\section{Ethics approval}

Not applicable

\section{Consent to participate}

Not applicable

\section{Consent for publication}

Not applicable

\section{Authors' contribution}

SD, PP, SC, PC performed the experiments and analyzed the results. AD analyzed the results and helped in writing the manuscript. DM and PT conceived the idea, designed the experiments, analyzed the results and wrote the manuscript.

\section{References}

Bergamini CM, Gambetti S, Dondi A, Cervellati C (2004) Oxygen, reactive oxygen speciesand tissue damage. Curr Pharm Des 10(14): 1611-1626

Chakraborty P, Joardar S, Ray S, Biswas P, Maiti D, Tribedi P (2018a) 3, 6-Di (pyridin-2-yl)-1, 2, 4, 5-tetrazine (pytz)-capped silver nanoparticles (TzAgNPs) inhibit biofilmformation of Pseudomonas aeruginosa: a potential approach toward breaking the wall ofbiofilm through reactive oxygen species (ROS) generation. Folia Microbiol 63(6): 763-772 
Chakraborty P, Daware AV, Kumari M, Chatterjee A, Bhattacharyya D, Mitra G, Akhter Y,Bhattacharjee S, Tribedi P (2018b) Free tryptophan residues inhibit quorum sensing of Pseudomonas aeruginosa: a potential approach to inhibit the development of microbialbiofilm. Arch Microbiol 200(10): 1419-1425

Chakraborty P, Tribedi P (2019) Functional diversity performs a key role in the isolation ofnitrogen-fixing and phosphate-solubilizing bacteria from soil. Folia Microbiol 64(3): 461-470

Chakraborty P, Dastidar DG, Paul P, Dutta S, Basu D, Sharma SR, Basu S, Sarker RK, SenA, Sarkar A, Tribedi P (2020) Inhibition of biofilm formation of Pseudomonas aeruginosaby caffeine: a potential approach for sustainable management of biofilm. Arch Microbiol 202(3): 623-635

Cortés ME, Bonilla JC, Sinisterra RD (2011) Biofilm formation, control and novel strategiesfor eradication. Sci Against Microbial Pathog Commun Curr Res Technol Adv 2: 896-905

Cowan MM (1999) Plant products as antimicrobial agents. Clin Microbiol Rev 12(4): 564-582

Crossley KB, Jefferson KK, Archer GL, Fowler VG (2009) Staphylococci in human disease,2nd illustrated edn.

Das MC, Paul S, Gupta P, Tribedi P, Sarkar S, Manna D, Bhattacharjee S (2016)3-Amino-4aminoximidofurazan derivatives: small molecules possessing antimicrobial andantibiofilm activity against Staphylococcus aureus and Pseudomonas aeruginosa. J Appl Microbiol 120(4): 842-859

Davies D (2003) Understanding biofilm resistance to antibacterial agents. Nat Rev Drug Discov 2(2): 114122

Daniels R, Vanderleyden J, Michiels J (2004) Quorum sensing and swarming migration inbacteria. FEMS Microbiol Rev 28(3): 261-289

Dewasthale S, Mani I, Vasdev K (2018) Microbial biofilm: current challenges in health careindustry. J Appl Biotechnol Bioeng 5:156-160

Dwivedi S, Wahab R, Khan F, Mishra YK, Musarrat J, Al-Khedhairy AA (2014) Reactiveoxygen species mediated bacterial biofilm inhibition via zinc oxide nanoparticles and theirstatistical determination. PloS one 9(11): e111289

Fukumura H, Sato M, Kezuka K, Sato I, Feng X, Okumura S, Fujita T, Yokoyama U, EguchiH, Ishikawa Y, Saito T (2012) Effect of ascorbic acid on reactive oxygen species productionin chemotherapy and hyperthermia in prostate cancer cells. J Physiol Sci 62(3): 251-257

Gupta P, Sarkar S, Das B, Bhattacharjee S, Tribedi P (2016) Biofilm, pathogenesis andprevention-a journey to break the wall: a review. Arch Microbiol 198(1): 1-15 
Hurlow J, Couch K, Laforet K, Bolton L, Metcalf D, Bowler P (2015) Clinical biofilms: achallenging frontier in wound care. Adv Wound Care 4(5): 295-301

Kaito C, Sekimizu K (2007) Colony spreading in Staphylococcus aureus. J Bacteriol 189(6):2553-2557

Kumar L, Chhibber S, Harjai K (2013) Zingerone inhibit biofilm formation and improveantibiofilm efficacy of ciprofloxacin against Pseudomonas aeruginosa PA01. Fitoterapia 90:73-78

Lowry OH, Rosebrough NJ, Farr AL, Randall RJ (1951) Protein measurement with the Folinphenol reagent. J Biol Chem 193: 265-275

Merritt PM, Danhorn T, Fuqua C (2007) Motility and chemotaxis in Agrobacterium tumefaciens surface attachment and biofilm formation. J Bacteriol 189(22): 8005-8014

Moreira CS, Silva ACJA, Novais JS, SáFigueiredo AM, Ferreira VF, da Rocha DR, CastroHC (2017) Searching for a potential antibacterial lead structure against bacterial biofilmsamong new naphthoquinone compounds. J Appl Microbiol 122(3): 651-662

Mukherjee K, Tribedi P, Mukhopadhyay B, Sil AK (2013) Antibacterial activity of long-chain fatty alcohols against mycobacteria. FEMS Microbiol Lett 338(2): 177-183

Paul P, Chakraborty P, Chatterjee A, Sarker RK, Dastidar DG, Kundu T, Sarkar N, Das A,Tribedi P (2020) 1, 4Naphthoquinone accumulates reactive oxygen species in Staphylococcus aureus. a promising approach towards effective management of biofilmthreat. Arch Microbiol 203(3): 1183-1193

Quijia CR, Chorilli M (2020) Characteristics, biological properties and analytical methods of piperine: A review. Crit Rev Anal Chem 50(1):62-77

Tribedi P, Gupta AD, Sil AK (2015) Adaptation of Pseudomonas sp. AKS2 in biofilm onlow-density polyethylene surface: an effective strategy for efficient survival and polymerdegradation. Bioresour Bioprocess 2(1): 1-10

Tsompanidou E, Sibbald MJ, Chlebowicz MA, Dreisbach A, Back JW, van Dijl JM, Buist G,Denham EL (2011) Requirement of the agr locus for colony spreading of Staphylococcus aureus. J Bacteriol 193(5): $1267-1272$

\section{Figures}




\section{Figure 1}

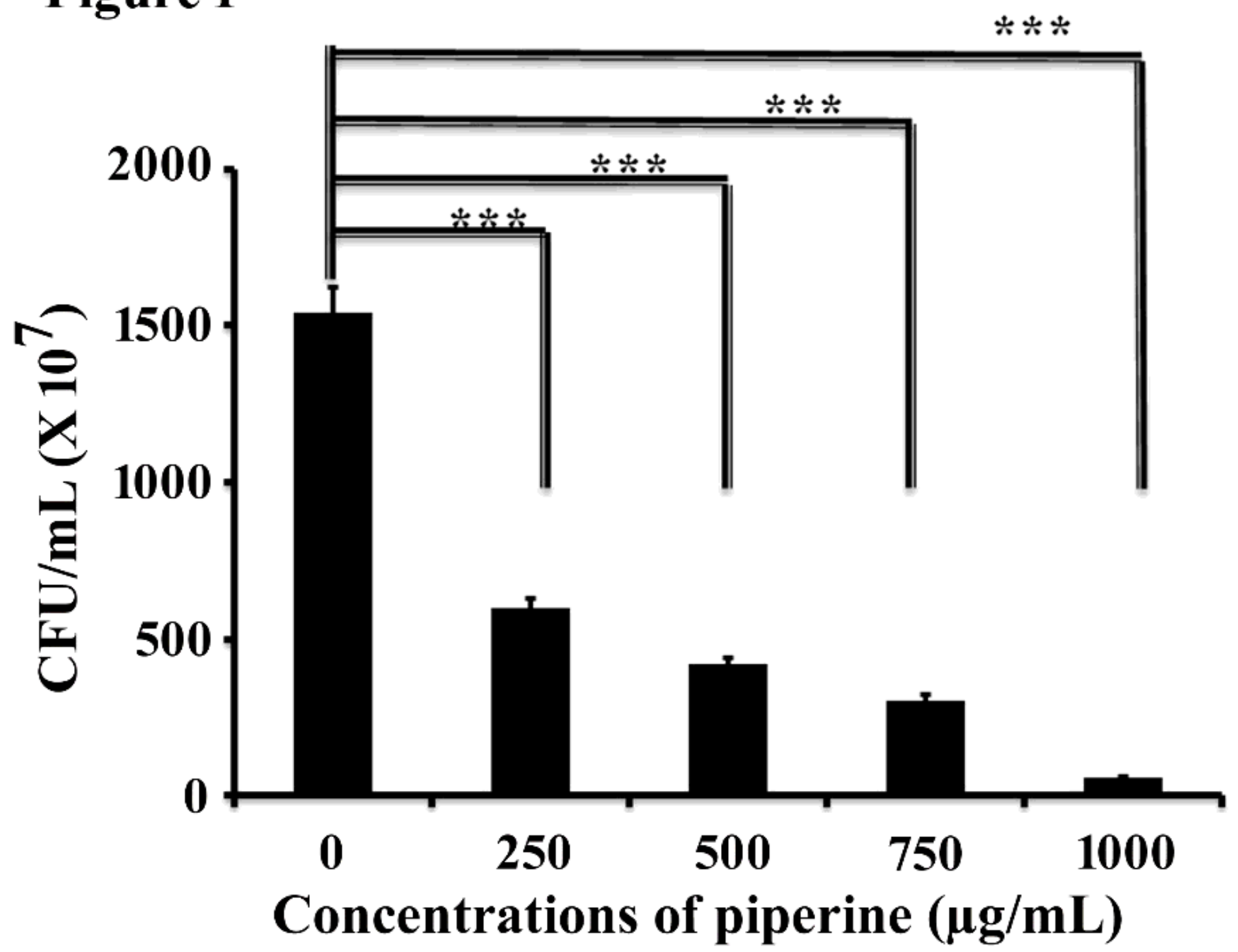

Figure 1

Higher concentrations of piperine exhibited significant antimicrobial activity against Staphylococcus aureus. Viable microbial counts were determined in both piperine treated and untreated growth media by performing the CFU assay under similar conditions as described in the Material and methods section. Each of the experiments was performed thrice. The result happened to be the average of the three experiments. Error bars signified the standard error of the mean. $P$ values were incorporated after statistical analysis using ANOVA. P values $<0.001$ was marked with ( $\left.{ }^{\star \star *}\right)$ to express the contrast in the data to control. 


\section{Figure 2}

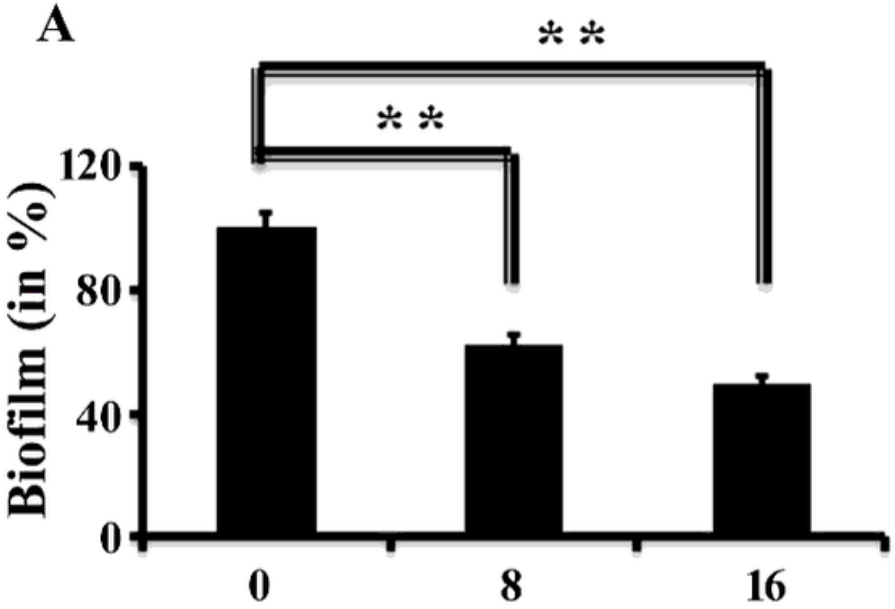

Piperine concentration $(\mu \mathrm{g} / \mathrm{mL})$

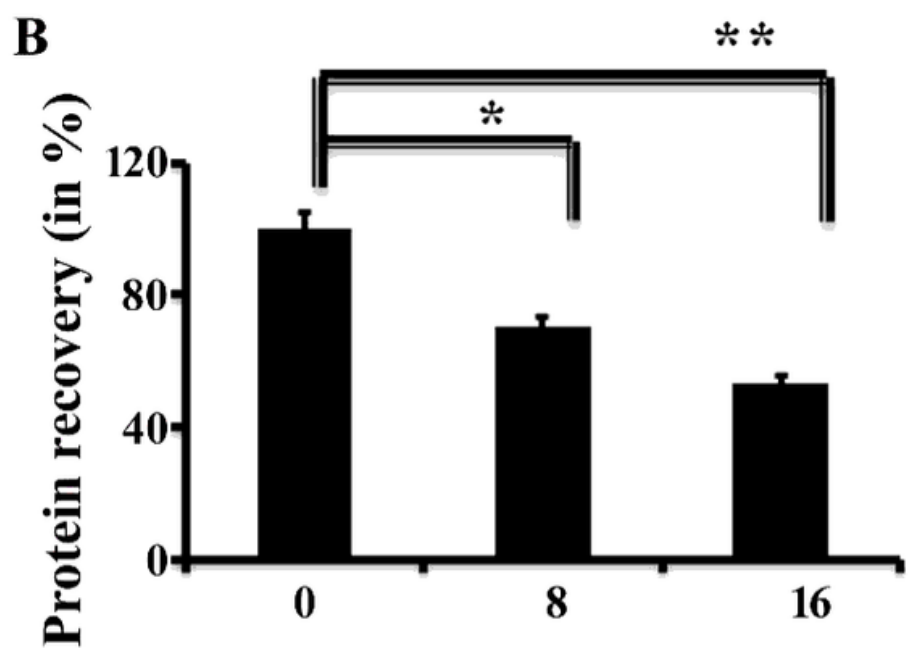

Piperine concentration $(\mu \mathrm{g} / \mathrm{mL})$

C Fluorescence microscopic image

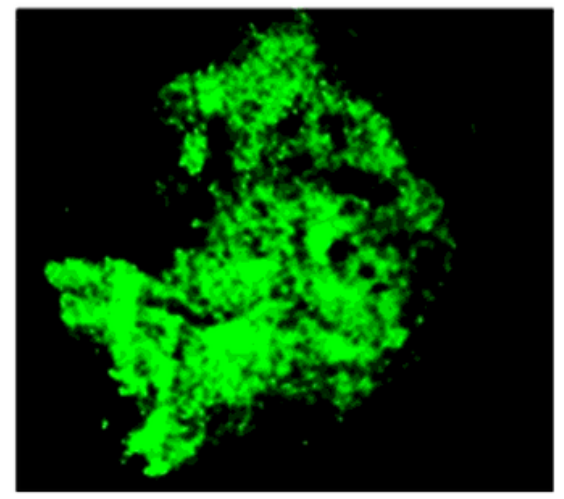

0

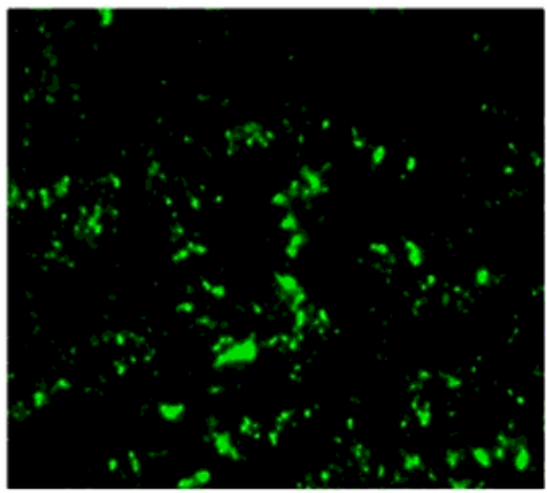

8

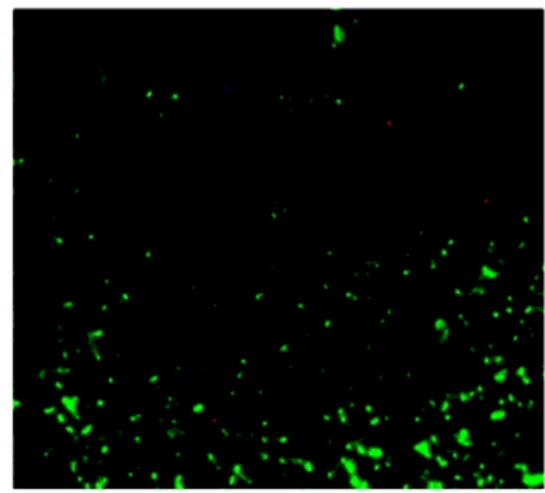

16

Piperine concentration $(\mu \mathrm{g} / \mathrm{mL})$

Figure 2

The tested concentrations of piperine showed efficient antibiofilm activity against Staphylococcus aureus. An equal number (1X105 CFU/mL) of cells were individually inoculated in sterile LB media challenged with different concentrations of piperine. In the control set, the cells were left untreated with piperine. All the experimental sets were incubated at $37^{\circ} \mathrm{C}$ for $24 \mathrm{~h}$. Post incubation, planktonic cells were removed and tubes were air-dried. Then, the CV assay (A) and total biofilm protein assay (B) were diligently performed as per the methodology described in the material and methods section. The result revealed the average of three experiments. Error bars signified the standard error of the mean. $P$ values were incorporated after statistical analysis using ANOVA. P values $<0.05$ were marked with $(*)$, P values $<$ 0.01 was marked with ${ }^{* *}$ ) to express the contrast in the data to the control set. (C) Fluorescence microscopic observation. To analyze the difference in the degree of microbial colonization, coverslips were recovered from both piperine treated and untreated growth media after the desired time of 
incubation. The recovered coverslips were stained with acridine orange and viewed under a fluorescence microscope. The figure indicated the representative of the images taken from 20 different fields and three separate experiments.

\section{Figure 3}

A

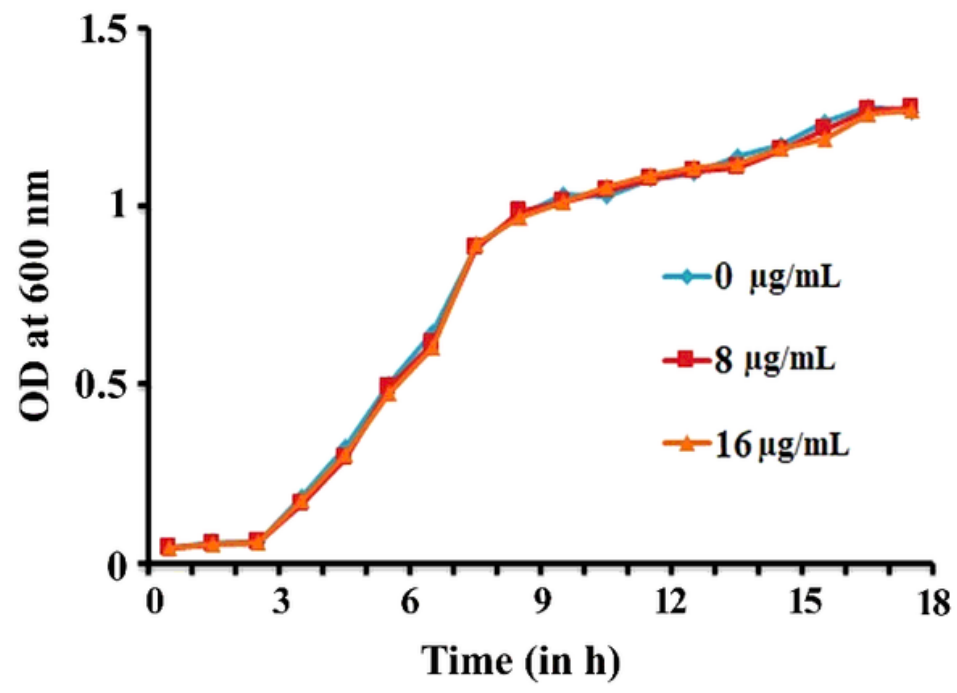

D

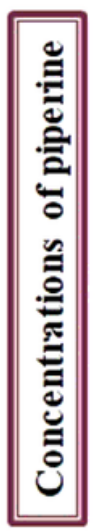

B

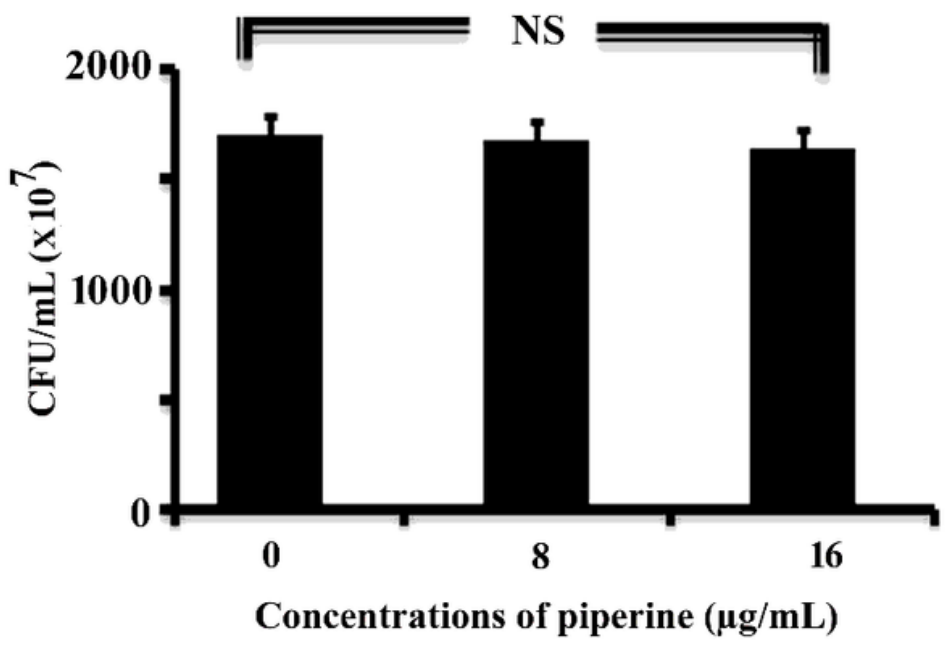

C

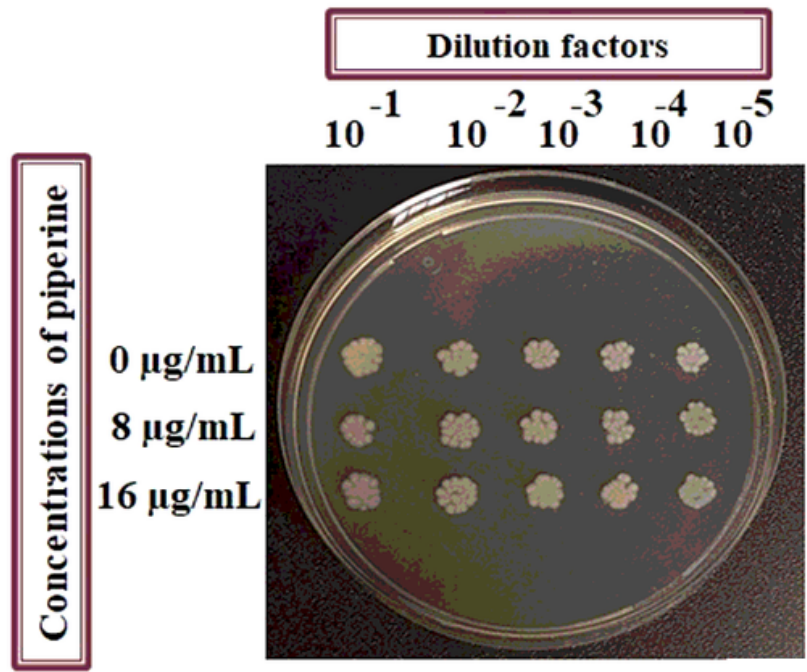

\section{Figure 3}

The lower concentrations of piperine did not exhibit any antimicrobial activity against Staphylococcus aureus. (A) Microbial growth curve analysis. A similar number of Staphylococcus aureus was allowed to grow separately in glass conicals containing $100 \mathrm{~mL}$ of sterile LB media. In the control set, the organisms were grown without exposure to piperine. All the experimental sets were incubated at $370 \mathrm{C}$. At regular time intervals, an equal volume of microbial cultures was collected from the respective growth media and its optical density was recorded at $600 \mathrm{~nm}$. (B) Viable microbial count analysis. An equal volume of microbial cultures was individually collected from both piperine treated and untreated growth media followed by determining the CFU count on sterile LB agar plates. Each of the experiments was performed thrice. Error bars signified the standard error of the mean. P values were incorporated after statistical 
analysis using ANOVA. P values above 0.05 were marked as N.S (Non-significant) to the control. (C) Microbial spot analysis. An equal volume of microbial cultures was separately collected from both piperine treated and untreated growth media followed by performing the serial dilution of the same. After that, $5 \mu \mathrm{L}$ sample collected from each dilution of both piperine treated and untreated growth media was separately spotted on a sterile LB agar plate. Then, the spotted LB agar plate was incubated for $24 \mathrm{~h}$ at $37^{\circ} \mathrm{C}$. (D) Clear zone analysis. A microbial lawn of Staphylococcus aureus was prepared by spreading the culture of the organism over the sterile LB agar plate. Wells were punctured on the microbial lawn wherein DMSO was added in one of the wells and piperine was loaded in the other two. After that, the plate was incubated for $24 \mathrm{~h}$ at $37^{\circ} \mathrm{C}$ to see the development of the clear zone (if any) around the wells.

\section{Figure 4}

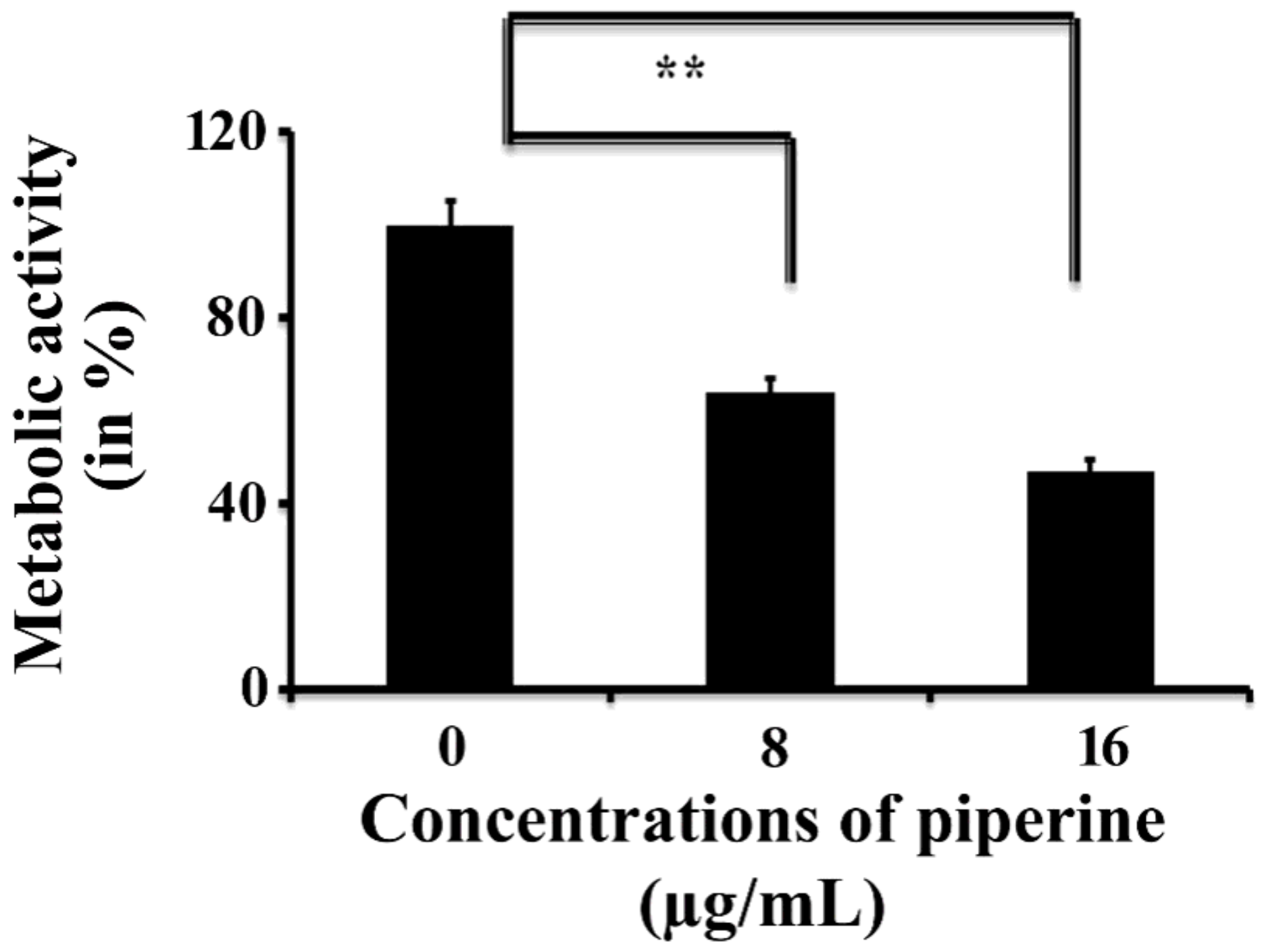

Figure 4

Piperine exhibited a considerable reduction in microbial metabolic activity. An equal number of cells were allowed to grow in sterile LB media either challenged with piperine or left unexposed. All the experimental 
sets were incubated at $370 \mathrm{C}$ for $24 \mathrm{~h}$. Post incubation, FDA hydrolysis assay was carried out in both piperine treated and untreated growth media. Each of the experiments was performed thrice. Error bars signified the standard error of the mean. $P$ values were incorporated after statistical analysis using ANOVA. $P$ values $<0.01$ was marked with $(* \star)$ to express the contrast in the data to the control set.

\section{Figure 5}

A

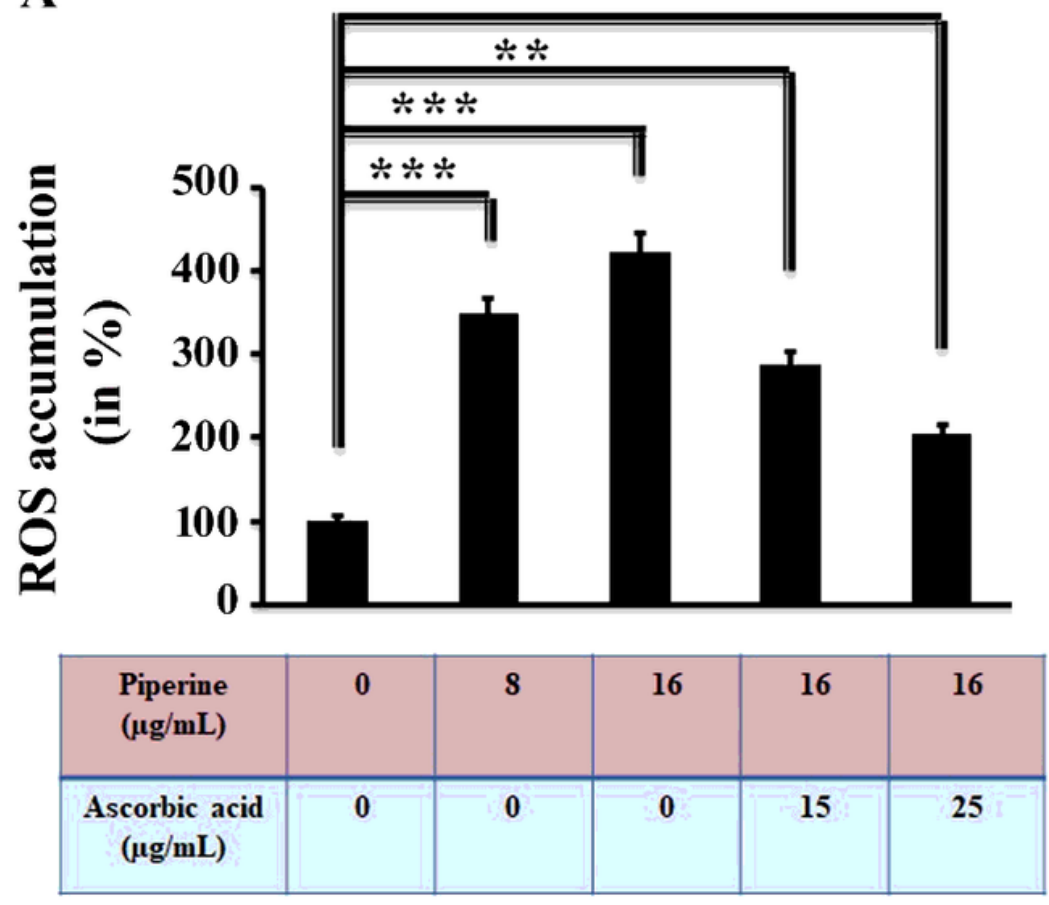

B

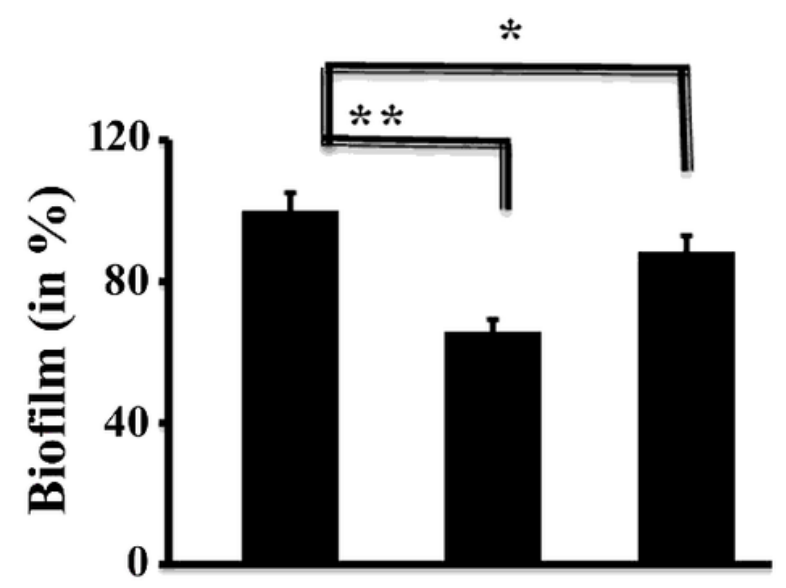

\begin{tabular}{|c|c|c|c|}
\hline $\begin{array}{c}\text { Piperine } \\
(\mu \mathrm{g} / \mathrm{mL})\end{array}$ & 0 & 16 & 16 \\
\hline $\begin{array}{c}\text { Ascorbic acid } \\
(\mu \mathrm{g} / \mathrm{mL})\end{array}$ & 0 & 0 & 25 \\
\hline
\end{tabular}

\section{Figure 5}

ROS generated under the influence of piperine inhibited the microbial biofilm formation considerably. (A) ROS profile. Cellular accumulation of ROS was measured in both piperine treated and untreated microorganisms by following the DCFDA based assay kit as mentioned in the material and methods section. (B) Biofilm profile. Microbial biofilm formation in both piperine treated and untreated growth media was determined by following the $\mathrm{CV}$ assay. Each of the experiments was performed three times. The result represented the average of the three. Error bars signified the standard error of the mean. $P$ values $<0.05$ was marked with (*), $P$ values $<0.01$ was marked with $(* *)$, and $P$ values $<0.001$ was marked with (***) in comparison to control. 


\section{Figure 6}

A

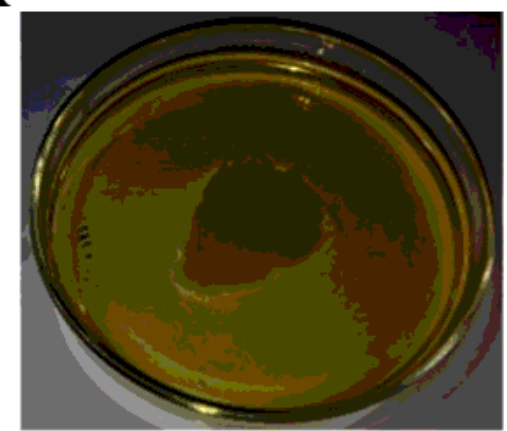

0

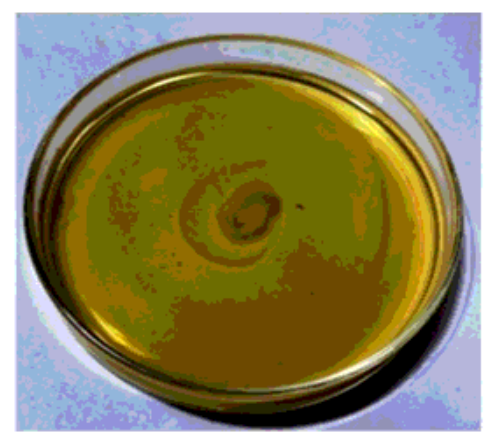

8

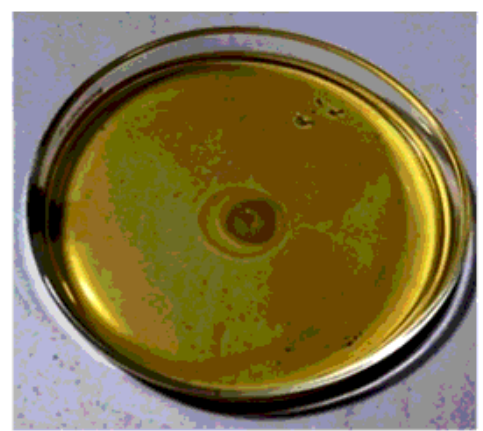

16

\section{Piperine concentrations \\ $(\mu \mathrm{g} / \mathrm{mL})$}

B

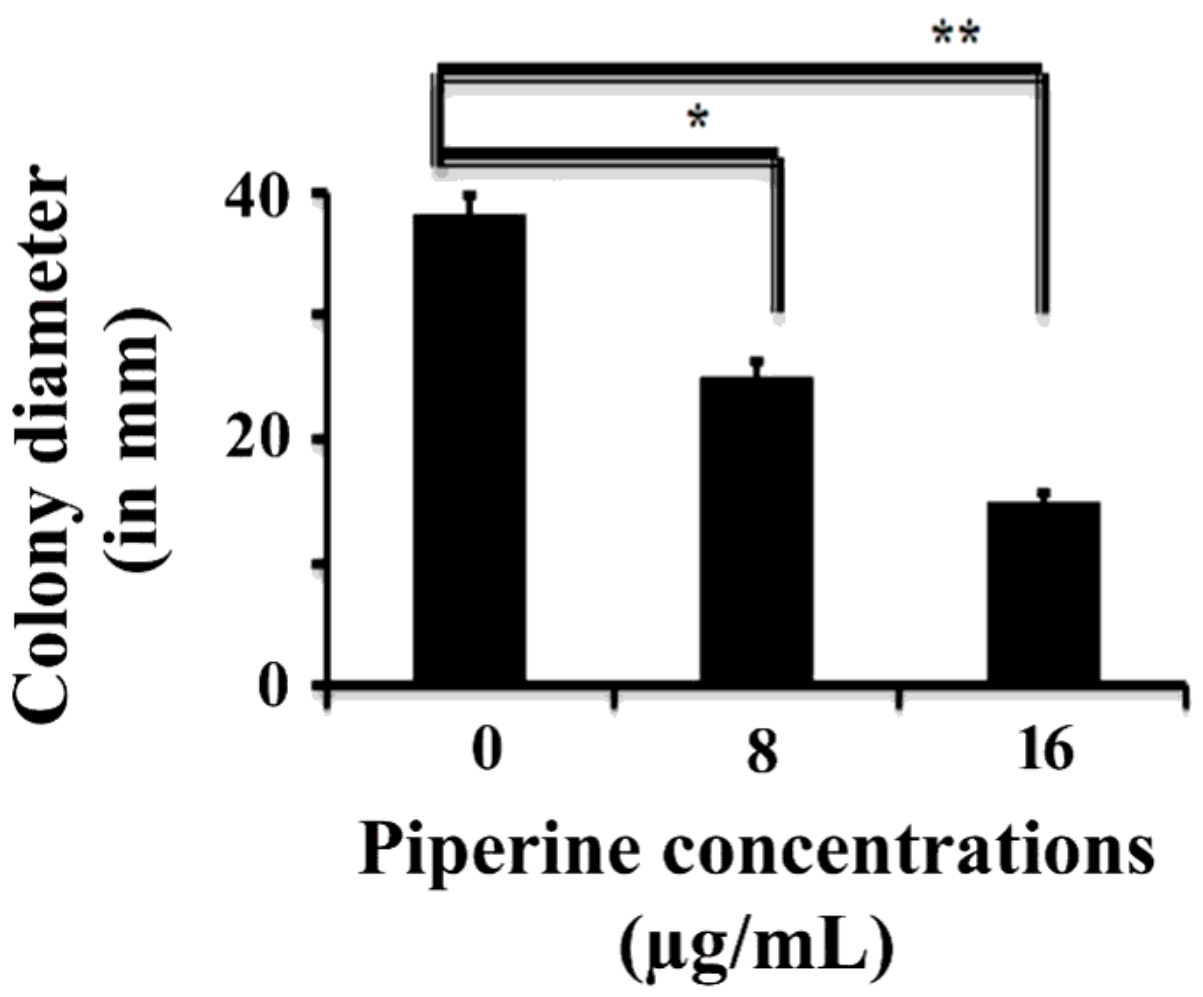

Figure 6

Piperine exhibited a considerable reduction in microbial motility. A similar number $(\sim 1 \mathrm{X} 105 \mathrm{CFU} / \mathrm{mL})$ of cells were independently recovered from both piperine treated and untreated growth media and individually spotted on the plates carrying sterile TSB supplemented with glucose $(5 \mathrm{gL}-1)$ and agar (8 gL1). Plates were then incubated at $37^{\circ} \mathrm{C}$ for $24 \mathrm{~h}$. Post incubation, the microbial motility was analyzed (A) and the zone of colony diameter $(B)$ was measured in $(\mathrm{mm})$. The result represented the average of three 
observations. Error bars happened to be mean \pm standard error of the mean. ANOVA was used to perform the statistical analysis. The P values $<0.05$ were marked with $\left({ }^{*}\right)$, and $<0.01$ was marked with $\left({ }^{\star *}\right)$ that showed a significant difference in contrast to the control. 